\title{
Principles of Equivalence: Their Role in Gravitation Physics and Experiments that Test Them*
}

\author{
Mark P. Haugan ${ }^{1}$ and C. Lämmerzahl ${ }^{2}$ \\ ${ }^{1}$ Purdue University, West Lafayette, IN 47907, USA \\ ${ }^{2}$ Department of Physics, University of Konstanz, Fach M674, 78457 Konstanz, Germany
}

June 1, 2018

\begin{abstract}
Modern formulations of equivalence principles provide the foundation for an efficient approach to understanding and organizing the structural features of gravitation field theories. Since theories' predictions reflect differences in their structures, principles of equivalence also support an efficient experimental strategy for testing gravitation theories and for exploring the range of conceivable gravitation physics. These principles focus attention squarely on empirical consequences of the fundamental structural differences that distinguish one gravitation theory from another. Interestingly, the variety of such consequences makes it possible to design and perform experiments that test equivalence principles stringently but do so in markedly different ways than the most familiar experimental tests.
\end{abstract}

\section{Equivalence Principles and the Structure of Gravitation Theo- ries}

\subsection{From the weak to Einstein's Equivalence principle}

Since the time of the Renaissance observations indicating that bodies fall in a gravitational field in a way that is independent of their internal composition and structure have been considered remarkable. Clearly Newton thought so since he deemed it necessary to perform pendulum experiments to verify this property of freefall as precisely as he could before he published laws of motion and universal gravitation that predict it [国. Einstein also found this property of freefall remarkable. The insight he gained by reflecting on it in his famous elevator Gedanken experiment [2] is communicated by what we now call the Einstein equivalence principle (EEP).

Einstein noted that if all bodies fall in the same way in an external gravitational field, an observer in freefall will find that freely falling bodies in his or her neighborhood move with uniform velocities relative to him or her and that the physics of pure particle mechanics in that neighborhood is indistinguishable from mechanics in the absence of gravity. This led him to suggest that a freely falling observer might find all other nongravitational physics in his or her neighborhood to be indistinguishable from such physics in the absence of gravity. Einstein then proceeded to show that if this were true for electrodynamic physics, which was all fundamental physics at the time, light propagating out of a gravitational potential well must suffer a redshift.

The following modern formulation of the EEP expresses the possibility suggested by Einstein in 1907. It states that the outcome of any local, nongravitational test experiment is independent of the experimental apparatus' velocity relative to the gravitational field and is independent of where and when in the gravitational field the experiment is performed. This captures Einstein's suggestion because, in principle, local nongravitational test experiments can be performed in spacetime regions

*Appeared in C. Lämmerzahl, C.W.F. Everitt, F.W. Hehl (eds.): Gyrod, Clocks, Interferometers...: Testing Relativistic Gravity in Space, Springer-Velag 2001 
where gravity is negligible. The two conditions that the EEP imposes are referred to as local Lorentz and local position invariance, respectively. Note that a local experiment is one performed within a spacetime region so small that the experimental apparatus can detect no tidal effects. A test experiment is one performed with an apparatus having a mass so small that the apparatus can detect no effect of the perturbation it induces in the gravitational field.

From the perspective of 1907, the EEP is a striking generalization from the observed equality of test body accelerations in a gravitational field, and, as a matter of history, the gravitational redshift was the first physical consequence to be derived on the assumption that this generalization is valid. Given this history, it is not surprising that refinements of experimental tests of the universality of freefall acceleration and of measurements of the gravitational redshift remain among the most widely recognized tests of the validity of the EEP. Efforts to refine both kinds of test continue today. Readers can refer to Lute Maleki's article [3] in this volume for details on a proposed space-based variation on gravitational redshift measurements.

Tests of the universality of free fall acceleration are often referred to as Eötvös experiments because of the classic torsion-balance version performed by Baron von Eötvös and collaborators early in this century [4]. As noted above, the process of refining such tests continues today. For example, the group of Adelberger at the University of Washington [5] recently reported results of a torsion-balance experiment that include the conclusion that the gravitational accelerations of beryllium and copper test bodies toward the Earth are equal to better than 2.5 parts in $10^{12}$. The ultimate refinement of tests of the equality of such accelerations may well be represented by the space-based STEP experiment under development at Stanford University. Readers can refer to an article in this volume for details on STEP [6].

Interest in such experiments remains high because our understanding of their significance as tests of the EEP has evolved significantly since 1907. The clear distinction Einstein made at that time between particle mechanics and other nongravitational physics, specifically electromagnetic physics, is no longer viable. We now understand how test bodies are composed of atoms and that they, in turn, are composites of the mass-energy of nucleons and electrons and of the electromagnetic, weakand strong-interaction binding energies of these particles. Consequently, the freefall acceleration of test bodies can be influenced by many, if not all, aspects of nongravitational physics in an external gravitational field, and experiments which test the universality of such accelerations turn out to be more profound tests of the EEP than one could have realized in 1907. We will return to this point later in section 2.1 .

Set aside, for a moment, the issue of whether or not the EEP is valid and questions regarding the precision to which we may be able to establish experimentally that it is valid. Is it possible to formulate a gravitation field theory which predicts that it is valid? It took Einstein almost ten years from the time of his 1907 insight to establish that the answer to this question is yes. He did so by formulating general relativity [7]. Interested readers can refer to the recent review by Norton [8] for a discussion of the fascinating and in many ways still controversial history of Einstein's development of general relativity and of others' early attempts to understand the theory. This story is plagued by many formulations of the equivalence principle, confusion regarding the significance of coordinates and covariance and so on. We will not delve further into it here. Instead, we discuss the kind of analysis one must do to determine whether or not any given gravitation field theory predicts the validity of the EEP.

\subsection{Theoretical contexts for analyses of the EEP}

If an analysis reveals outcomes of some local nongravitational test experiment that depend on the velocity of the experimental apparatus relative to an external gravitational field or on where or when in that field the experiment is performed, it is clear that the underlying theory is nonmetric, that is, it violates the EEP. Significantly, such an analytical result also provides the basis for actual experiments that search for the specific preferred-frame or preferred-location effect revealed by the analysis. This approach has led to the development of many stringent new tests of the EEP that are 
quite different from familiar Eötvös experiments and gravitational redshift measurements. It has also clarified which structural features of gravitation field theories are constrained by experimental evidence that the EEP is valid to some level of precision.

Lagrangian field theory provides a natural setting for a general discussion of gravitation field theories. However, we note that much of what follows can be discussed in terms of gravitation field equations and matter-field equations of motion. Indeed, a number of recent papers exploit this latter approach to consider modifications of the Maxwell equations caused by quantum gravity effects. Papers by Gambini and Pullin [9] and by Ellis et al. [10] are examples. The paper of Haugan and Lämmerzahl [11] begins the analysis of physical consequences of a broad range of conceivable Maxwell equation modifications of this kind and the consideration of experiments that could detect them or constrain their magnitudes.

Returning to Lagrangian-based gravitation theories, we recall that each admits a formulation via an action principle,

$$
\delta \int \mathcal{L}\left(\psi_{g}, \psi_{m}\right) d^{4} x=0
$$

Here, $\psi_{g}$ denotes dependence of the Lagrangian density on gravitational potentials and their derivatives, and $\psi_{m}$ denotes dependence on matter fields and their derivatives. Not that long ago, one would have restricted attention to theories in which field derivatives appear only in conventional "kinetic" terms in $\mathcal{L}$, but attitudes have changed so that field theories tend to be viewed as effective rather than fundamental theories. Consequently, there is now a greater willingness to consider dependence on higher-order derivatives and the presence of derivative couplings between fields. Such things can make theories nonrenormalizable, but this is not the issue for effective theories that it is for fundamental ones.

A theory's Lagrangian density $\mathcal{L}$ can be split into a purely gravitational part and "nongravitational" remainder, $\mathcal{L}=\mathcal{L}_{g}+\mathcal{L}_{n g}$. The gravitational part $\mathcal{L}_{g}$ depends only on gravitational potentials and their derivatives. Its form specifies the dynamics of free gravitational fields in the theory. The nongravitational part $\mathcal{L}_{n g}$ depends on gravitational potentials and their derivatives and on matter fields and their derivatives. Its form specifies the coupling between matter and gravity in the theory. Its form determines both how matter responds to gravity and how matter acts as a source of gravity.

\subsection{The role of locality}

The matter fields involved in a local, nongravitational test experiment do not perturb gravitational potentials to a degree that the experiment can detect. It follows that when using a theory to predict the outcome of such an experiment we can treat gravitational potentials as specified functions which represent the gravitational environment generated by some source. They are external potentials. To predict the outcome of the experiment one needs only a theory's equations which govern the evolution of matter fields in the relevant gravitational environment. We derive these gravitationally-modified equations of motion from the theory's action principle (1) by considering variations of the matter fields while keeping the external gravitational potential functions fixed in the appropriate form. Consequently, the outcomes of experiments that directly test the EEP depend on the form of a theory's nongravitational Lagrangian density $\mathcal{L}_{n g}$ alone. Clearly, evidence that the EEP is valid to some precision can constrain only the manner in which matter couples to gravity.

Since experiments that directly test the EEP are local as well as test experiments, their outcomes are insensitive to the global form of external gravitational potentials. It is sufficient to consider initial terms of the Taylor-series expansions of external potentials when using a theory's action principle to predict a local experiment's outcome. The expansions should be made about an event inside the experimental apparatus during the course of the experiment. Keeping terms through first order is sufficient to predict the outcome of any local experiment. However, in some important cases it is sufficient to keep only zeroth-order terms. In particular, this can be the case when an experiment is completed quickly enough that the experimental apparatus can detect no effect of external potential 
time dependence and no effect of accelerations induced by external potential spatial dependence. This is generally true of realistic local experiments which measure atomic transition frequencies, for example, and explains why atomic clocks may generally be treated as realizations of ideal clocks in the sense defined by theories of relativity.

To make the remainder of this discussion a bit more concrete focus on experiments involving such atomic transitions. To this point, we have established that a theory's predictions of their outcomes follows from the form that its nongravitational Lagrangian density $\mathcal{L}_{n g}$ takes when values of the external gravitational potentials and, if there are derivative matter-gravity couplings, values of their derivatives at an event inside the experimental apparatus during the experiment are plugged in. The resulting Lagrangian density has no explicit dependence on the spacetime coordinates and involves only matter fields. It determines the gravitationally-modified equations of motion which govern the structure of atoms treated as local test bodies. Note, however, that if we are interested only in atomic transition frequencies, we need not deal with these equations. We can, instead, compute energies of atomic states directly.

\subsection{Relevant observables}

An expression for such energies follows from the form of the Lagrangian density introduced in the preceding paragraph because it is time independent. If natural coordinates are used in representing a theory's action principle (1), the energy expression's form will be that of the Standard Model Hamiltonian plus perturbating terms. In this context, natural coordinates are ones in which the form of the representation of the theory's nongravitational Lagrangian density $\mathcal{L}_{n g}$ reduces to the familiar representation of the Standard Model Lagrangian density as gravity is "turned off." Schwarzschild coordinates provide a familiar example of natural coordinates in the context of general relativity and situations in the external gravitational potential is static and spherically symmetric.

Accurate estimates of the energies of atomic states are easily computed when the gravitationallymodified Hamiltonian is a perturbed Standard Model Hamiltonian. In general, the results depend on an atom's velocity through and location in the external gravitational potentials. This is the case because the perturbing Hamiltonian terms reflect not only the form of the gravitation theory's nongravitational Lagrangian density $\mathcal{L}_{n g}$ but also the atom's gravitational environment. This environment is represented by the values in the atom's neighborhood of the external gravitational potentials and, if there are derivative matter-gravity couplings, the values of their derivatives.

Despite their velocity and location dependence these computed atomic state energies may not represent preferred-frame or preferred-location effects that signal violation of the Einstein equivalence principle. Even when we are using natural coordinates they are merely coordinate energies. Only velocity or location dependence of an experimentally measured atomic state energy, or frequency of a transition between such states, would constitute a genuine preferred-frame or preferred-location effect.

The distinction between coordinate energies or frequencies and measured energies or frequencies is, in some respects, subtle. However, it is not difficult to appreciate if one remembers that, fundamentally, any measurement is simply the comparison of a property of some system of interest to the corresponding property of a chosen standard system. Thus, a measurement of the frequency of a transition between some pair of atomic states is simply a comparison of its coordinate frequency to the coordinate frequency of a selected standard transition. For example, one can imagine locking a laser to the transition whose frequency is to be measured and a second laser to the frequency of the standard transition. The coordinate frequency of each laser can depend on velocity through or location in the external gravitational potential, but the relative or beat frequency between them, which is the measured frequency, may not. While it may seem far-fetched to imagine cases in which coordinate energies of all atomic states depend on velocity through or location in external gravitational potentials in precisely the same way, thus, causing such dependence to cancel from measured energies, this is precisely what metric theories of gravity like general relativity predict. The preferred-frame or preferred-location effects predicted by nonmetric theories of gravity occur 
because their matter-gravity couplings distinguish between the contributions of rest-mass and of different types of interaction-energy to the energies of atomic states and, so, prevent such universal cancellations of coordinate effects. If such couplings are present, the observable frequency ratio for a pair of atomic clocks whose ticking rates are governed by different atomic transition and which move together through an external gravitational potential can depend on the clocks' location in and velocity through the potential. In the limit of slow motion and weak gravitation this frequency ratio takes the form

$$
\frac{\nu_{1}(\vec{x}, \vec{v})}{\nu_{2}(\vec{x}, \vec{v})}=\frac{\nu_{1}^{0}}{\nu_{2}^{0}}\left(1+\alpha_{i j} v^{i} v^{j}+\beta_{i j} U^{i j}(\vec{x})\right)
$$

where the $x^{i}$ denote natural spatial coordinates that reduce to Cartesian ones as gravity is turned off, the $v^{i}$ denote corresponding components of the clocks' coordinate velocity and $U^{i j}(\vec{x})$ denotes the usual Newtonian gravitational potential tensor at the clocks' location. The parameters $\alpha_{i j}$ and $\beta_{i j}$ depend on the particular transitions controlling the atomic clock rates, except in metric theories of gravity which predict that they all vanish. Their tensor character reflects the fact that the orientation of the atoms whose transitions govern the atomic clock rates can affect the observed frequency ratio (2). Nonvanishing $\alpha_{i j}$ and $\beta_{i j}$ parameters characterize preferred-frame and preferred-location effects, respectively.

To conclude this discussion of the way in which Lagrangian-based gravitation theories predict outcomes of local, nongravitational test experiments, briefly consider the acceleration of test bodies in an external gravitational field once more. We focus on the freefall of atoms since, as we noted earlier, realistic test bodies are simply assemblages of them.

The analysis of atomic systems outlined above yields an expression for the coordinate energy of any atom in any state of interest. This energy is a function of the atom's velocity relative to and location in an external gravitational potential

$$
E=m c^{2}+\frac{1}{2} m\left(\delta_{i j}+\frac{\delta m_{\mathrm{i} i j}}{m}\right) v^{i} v^{j}+m\left(\delta_{i j}+\frac{\delta m_{\mathrm{g} i j}}{m}\right) U^{i j}(\vec{x}),
$$

where $\delta m_{\mathrm{i} i j}$ and $\delta m_{\mathrm{g} i j}$ are the anomalous inertial and gravitational mass tensors. They depend on the particular state of the particular atom under consideration, except in metric theories of gravity which predict that they vanish. The $\alpha_{i j}$ and $\beta_{i j}$ parameters appearing in (2) are determined by the anomalous mass tensors of the states involved in the atomic clock transitions considered above.

When the external gravitational potential is time independent, the coordinate energy function (3) is globally conserved and its dependence on atomic velocity and location determines the atom's coordinate acceleration via familiar energy conservation arguments,

$$
a^{i}=\delta^{i j} \partial_{j} U+\frac{\delta m_{\mathrm{i}}^{i j}}{m} \partial_{j} U+\delta^{i j} \frac{\delta m_{\mathrm{g} k l}}{m} \partial_{j} U^{k l}(\vec{x}),
$$

(here $\delta m_{\mathrm{i}}^{i j}=\delta m_{\mathrm{i} i j}$ ). Kenneth Nordtvedt [12] and Mark Haugan 13] exploit such arguments to relate the outcomes Eötvös experiments to the outcomes of gravitational redshift measurements and other tests of the EEP.

In the end, the preceding overview of the kind of analysis one must do to determine the outcomes of local nongravitational test experiments predicted by Lagrangian-based gravitation field theories brings one full circle. We have come back to the most familiar experimental tests of the EEP, but with a deeper appreciation of the significance of their results. In the next section we consider examples of nonmetric theories and formalisms encompassing whole classes of such theories within which preferred-frame and preferred-location effects have been analyzed to provide a basis for testing the EEP. Much of the work on tests of the EEP done before 1993 is thoroughly reviewed in the early chapters of Clifford Will's Theory and Experiment in Gravitation Physics [14, for an update see [15], see also 16]. 


\section{Theoretical frameworks for the analysis of EEP tests}

The approach outlined in the preceding section can be used to determine preferred-frame and preferred-location effects predicted by any nonmetric theory of gravity. Such effects reflect the form of the theory's nongravitational Lagrangian density $\mathcal{L}_{n g}$ once the external gravitational potential in which a local nongravitational test experiment is performed has been plugged in. Proceeding in this fashion, we would have to analyze and reanalyze any given experiment to determine its outcome as predicted by competing theories.

It is, instead, more efficient to analyze local nongravitational test experiments once and for all within the context of more general theoretical frameworks that encompass broad classes of nonmetric gravitation theories and gravitational environments. Such frameworks are based on models of the nongravitational Lagrangian or corresponding matter field equations that depend on phenomenological gravitational potentials in ways that encompass the forms of these structures in many nonmetric theories and environments. The outcome of an experiment predicted within such a framework immediately yields the outcome predicted by any nonmetric theory it encompasses when the framework's phenomenological gravitational potentials are expressed in terms of the particular theory's potentials. The efficiency of this approach is somewhat like that provided by the PPN formalism [14] when dealing with the gravitational dynamics of metric theories.

Analyses carried out in the general nonmetric frameworks discussed below have the additional benefit of identifying mechanisms that lead to preferred-frame or preferred-location effects in entire classes of nonmetric theories and of providing theory-independent parametrizations of such effects that are useful in discussing the results of experiments designed to search for them.

\subsection{The $T H \epsilon \mu$-formalism}

The TH $\epsilon \mu$-formalism (see [17, 14]) is based on the form of the Lagrangian governing the dynamics of point particles with mass $m_{i}$ and charge $q_{i}$ and of the electromagnetic field in a static, spherically symmetric background gravitational field described by the phenomenological gravitational potentials $T, H, \epsilon$ and $\mu$ :

$$
L=-\sum_{i} m_{i} \int \sqrt{T-H \dot{x}_{i}^{2}} d t+\sum_{i} q_{i} \int A_{a} \dot{x}_{i} d x^{a}+\frac{1}{8 \pi} \int\left(\epsilon \boldsymbol{E}^{2}-\frac{1}{\mu} \boldsymbol{B}^{2}\right) d^{4} x .
$$

A striking feature of this framework and the nonmetric theories it encompasses is that the limiting speed of massive particles in the neighborhood of some point in the gravitational field can differ from the speed of light there. These coordinate speeds are given, respectively, by the values of $\sqrt{T / H}$ and $1 / \sqrt{\epsilon \mu}$ at the point of interest. Preferred-frame effects result when the ratio of these speeds is not unity. Variation of the relative values of $T, H, \epsilon$ and $\mu$ with position in the gravitational field can also lead to preferred-location effects. Computations of the energies of atomic states using natural quantum mechanical extensions of the classical $T H \epsilon \mu$ Lagrangian reveal both kinds of effects and yield predictions for anomalous inertial and gravitational mass tensors (compare Eq.(3)) [13, 18, 19]. A quantum field theoretic extension of the formalism reveals EEP violations discernable in measurements of the Lamb shift, the anomalous magnetic moment of the electron and related phenomena [21].

This test theory has been widely used to interpret the results of experimental tests of the EEP. For example, its predictions of the energies of atomic states [13], [18] and [19] have be used to interpret Hughes-Drever type experiments as well as the Vessot-Levine rocket redshift experiment 20, 14.

Originally conceived as a framework for analyzing the physics of charged particles and electromagnetic fields in an external gravitational field, the $T H \epsilon \mu$-formalism has also been extended in a natural way to cover the other sectors of nongravitational physics comprising the Standard Model [22]. 


\subsection{The $\chi g$-formalism}

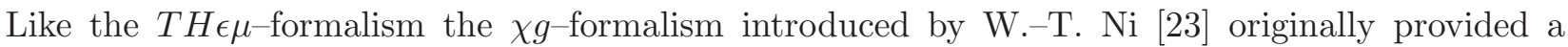
framework for the analysis of electrodynamic physics in a background gravitational field and has subsequently been extended to cover other sectors of the Standard Model. Unlike the TH $\epsilon \mu$-formalism the $\chi g$-formalism is not restricted to static, spherically symmetric gravitational environments. The $\chi$ of its name refers to a tensor field appearing in the electromagnetic part of the nongravitational Lagrangian density upon which the formalism is based,

$$
\mathcal{L}_{\mathrm{em}}=-\frac{1}{16 \pi} \chi^{\alpha \beta \gamma \delta} F_{\alpha \beta} F_{\gamma \delta}
$$

The independent components of this tensor comprise twenty-one phenomenological gravitational potentials capable of representing gravitational fields in a very broad class of nonmetric gravitation theories.

The coupling of one particular phenomenological potential to the electromagnetic field is interesting because it can be expressed as a purely derivative coupling to a pseudoscalar field $\varphi$. A particle physicist would describe it as an axion coupling. A relativist would describe it as a coupling to axial torsion [24]. The Hojmann-Rosenbaum-Ryan-Shepley theory [25] is but one example of a theory encompassed by Ni's $\chi g$-formalism. It features a novel torsion coupling that has been shown to predict effects inconsistent with the results of experimental tests of the weak equivalence principle.

\subsection{The Kostelecky Formalism}

String theory has the potential to provide a quantum theory of gravity that is unified with other fundamental theories of matter and interactions. Recently Colladay and Kostelecky have introduced a framework for treating the possibility of spontaneous breakdown of Lorentz symmetry in the context of string theory [31, 32]. While somewhat different from the sources of preferred-frame effects considered to this point, these string induced effects are considered here because they lead to modifications of the Dirac and Maxwell equations like those considered in the next subsection.

\subsection{Formalisms based on matter-field equations of motion}

The effects an external gravitational field on the dynamics of matter fields can be dealt with at the level of equations of motion rather than Lagrangians. A formalism based on forms of the equations of motion has the advantage of directly addressing the following natural requirements one would demand of the dynamics of any quantum field (i) deterministic evolution, (ii) the superposition principle, (iii) a finite propagation speed (whose maximum value, since it need not be isotropic, we call $c_{D}$ ) and (iv) the conservation of probability.

The equations governing the motion of a Dirac field which satisfy these requirements are a first-order hyperbolic system of the form

$$
0=i \widetilde{\gamma}^{\mu} \partial_{\mu} \varphi+M \varphi
$$

or, in $3+1$-form $(\hat{\mu}=1,2,3)$

$$
i \partial_{0} \varphi=c_{D} \widetilde{\alpha}^{\hat{\mu}} c \partial_{\hat{\mu}} \varphi+c_{D} \widetilde{\Gamma} \varphi+m c_{D}^{2} \widetilde{\beta} \varphi
$$

which we call a generalized Dirac equation $\left(\widetilde{\alpha}^{\hat{\mu}}=\left(\widetilde{\gamma}^{0}\right)^{-1} \widetilde{\gamma}^{\hat{\mu}}\right)$. The matrices $\widetilde{\gamma}^{\mu}$ are not assumed to define a Clifford algebra, instead they satisfy $\widetilde{\gamma}^{\mu} \widetilde{\gamma}^{\nu}+\widetilde{\gamma}^{\nu} \widetilde{\gamma}^{\mu}=2 g^{\mu \nu}+X^{\mu \nu}$ where $g^{\mu \nu}=\frac{1}{4} \operatorname{tr}\left(\widetilde{\gamma}^{\mu} \widetilde{\gamma}^{\nu}\right)$ and $X^{\mu \nu}$ is a matrix. In general, $M$ is also a matrix. A distinctive feature of this generalized Dirac equation is that it predicts a splitting of the null cones and mass shells. (For another modification of the Dirac equation see [26] in this volume.) 
Taking the non-relativistic limit and specifying a general position-dependence of the matrices $\widetilde{\gamma}^{\mu}$ and $M$, one derives the generalized Pauli equation [27]

$$
\begin{aligned}
i \frac{\partial}{\partial t} \varphi= & -\frac{1}{2 m}\left(\delta^{i j}-\frac{\delta m_{\mathrm{i}}^{i j}}{m}-\frac{\delta \bar{m}_{\mathrm{i} k}^{i j} \sigma^{k}}{m}\right) \partial_{i} \partial_{j} \varphi+\left(c_{D} A_{j}^{i}+\frac{1}{m} a_{j}^{i}\right) \sigma^{j} i \partial_{i} \varphi \\
& +\left[m U(\boldsymbol{x})+\boldsymbol{C} \cdot \boldsymbol{\sigma} m U(\boldsymbol{x})+\delta m_{\mathrm{g} i j} U^{i j}(\boldsymbol{x})+c_{D} \boldsymbol{T} \cdot \boldsymbol{\sigma}+m c_{D}^{2} \boldsymbol{B} \cdot \boldsymbol{\sigma}\right] \boldsymbol{\varphi}
\end{aligned}
$$

where the anomalous coefficients $\delta m_{\mathrm{i}}^{i j}, \delta \bar{m}_{\mathrm{i} k}^{i j}, A_{j}^{i}, a_{j}^{i}, \boldsymbol{C}, \delta m_{\mathrm{i}}^{i j}, \boldsymbol{B}$ stem from those parts of the $\widetilde{\gamma}-$ matrices which prevent them from defining a Clifford algebra and from anomalous terms in the mass matrix $M$, for example, $\frac{\delta m_{i}^{i j}}{m}+\frac{\delta \bar{m}_{i k}^{i j}}{m} \sigma^{k}=\frac{1}{2}(1+\beta) \widetilde{\alpha}^{(i} \widetilde{\alpha}^{j)}$ where $\beta$ is the usual Dirac $\gamma^{0}$ and $\sigma^{i}$ are the usual Pauli matrices. The generalized Pauli equation predicts preferred-frame and preferredlocation effects. Terms like those representing couplings between spin and the Newtonian gravitational potential were first introduced in references [28, 29] and [30].

The Pauli equation (9) is a generalization of Schroedinger equation provided by M . Haugan's approach [13] to the dynamics of scalar matter. As in the case of preceding formalisms, this Pauli equation provides a basis for broad range of experimental tests of the EEP, including experiments exploiting matter-wave interferometry. The classical limit of the generalized Pauli equation describes the free fall of classical spin-polarized bodies,

$$
a^{i}=\delta^{i j} \partial_{j} U+\left[\frac{\delta m_{\mathrm{i}}^{i j}}{m}+2\left(\frac{\delta \bar{m}_{\mathrm{i} k}^{i j}}{m}+\delta^{i j} C_{k}\right) S^{k}\right] \partial_{j} U+\delta^{i j} \frac{\delta m_{\mathrm{g} k l}}{m} \partial_{j} U^{k l}(\vec{x}) .
$$

Notice that not all of the anomalous parameters appearing in the quantum equation (9) survive in the classical freefall acceleration. Only by considering the evolution of the spin as well can one design experiments in the classical limit that are sensitive to all possible anomalies.

Once a generalized Dirac equation (8) is available we can address the dynamics of the electromagnetic field in an analogous way. The electromagnetic field can be defined operationally by considering the phase shifts in charged particle interferometry. Assuming that the dynamics of electromagnetic fields satisfies the same requirements as we demanded for the dynamics of the Dirac field, this leads to generalized Maxwell equations of the form,

$$
\partial_{[\mu} F_{\nu \rho]}=0, \quad 4 \pi j^{\mu}=\lambda^{\mu \nu \rho \sigma} \partial_{\nu} F_{\rho \sigma}+\bar{\lambda}^{\mu \rho \sigma} F_{\rho \sigma}
$$

In the case of small deviations from minimal coupling to the Riemannian space-time metric $g_{\mu \nu}$, we have $\lambda^{\mu \nu \rho \sigma}=\delta^{\mu[\rho} g^{\sigma] \nu}+\delta \lambda^{\mu \nu \rho \sigma}$ with small values of $\delta \lambda^{\mu \nu \rho \sigma}$ and $\bar{\lambda}^{\mu \rho \sigma}$. Clearly, $\delta \lambda^{\mu \nu \rho \sigma}$ can induce anistropic propagation of light and birefringence. The $\bar{\lambda}^{\mu \rho \sigma}$ can also modify propagation, in some cases leading to a damping of electromagnetic waves.

The generalized Dirac equation (8) and Maxwell equations (11) can be used just as the corresponding equations that emerge from the $T H \epsilon \mu$-formalism or the $\chi g$-formalism, respectively, to analyze to properties of atoms in background gravitational fields. They do, however, encompass a wider range of nonmetric couplings that influence spin and polarization. Consequently, they provide the broadest possible basis for the interpretation of experimental tests of the EEP.

\section{Motivations for continued testing of the EEP}

Although all tests of the EEP, including some of remarkable precision, have so far failed to detect any hint of a violation, recent theoretical developments continue to suggest the EEP must be violated at some level. All approaches to quantizing gravity and to unifying it with the other fundamental interactions currently under study are capable of predicting such violations. 


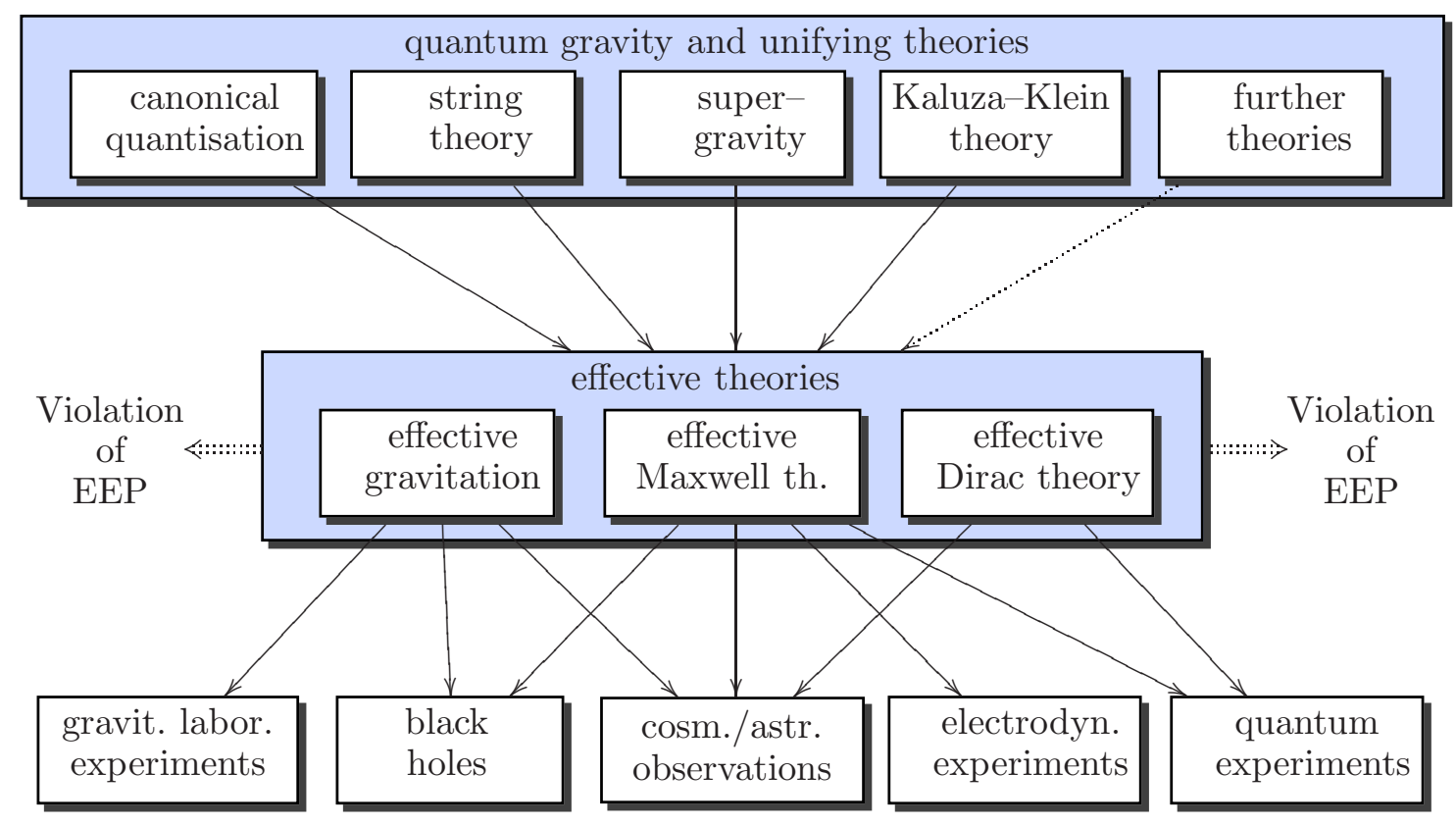

Figure 1: Sources of violations of the EEP and classes of experiments or observations that are sensitive to them.

\subsection{String theory}

Today, string theory is among the most promising candidate for a theory of quantum gravity fully unified with other fundamental interactions, and it has been shown to predict a variety of EEP violations.

For example, departures from universal free fall accelerations have been computed in references [33, 34]. The composition-dependent component of test-body acceleration is estimated to be as large as a part in $10^{15}$ of the mean gravitational acceleration. The proposed STEP experiment could easily detect such an anomaly (see [6]).

String theory can also predict a time-varying fine structure constant because of couplings to scalar (dilatonic) fields, for example, see 35.

In the latest versions of string theory, physical particles and fields are confined to the neighborhoods of $D$-branes and their propagation may be affected by recoil of the branes caused by that propagation [36]. The effect of this recoil can be accounted for via an energy-dependent effective metric. This leads to modifications of the Maxwell equations,

$$
\begin{aligned}
\nabla \cdot \boldsymbol{E}+\overline{\boldsymbol{u}} \cdot \partial_{t} \boldsymbol{E} & =0 & \boldsymbol{\nabla} \cdot \boldsymbol{B} & =0 \\
\nabla \times \boldsymbol{B}-\left(1-\bar{u}^{2}\right) \partial_{t} \boldsymbol{E}+\overline{\boldsymbol{u}} \times \partial_{t} \boldsymbol{B}+(\overline{\boldsymbol{u}} \cdot \boldsymbol{\nabla}) \boldsymbol{E} & =0 & \nabla \times \boldsymbol{E} & =-\partial_{t} \boldsymbol{B},
\end{aligned}
$$

which predict dispersive light propagation. Analogous modifications of the Dirac equation account for the effect of brane recoil on the propagation of neutrinos and other fermions [37],

$$
\gamma^{a} \partial_{a} \psi-m \psi+\gamma^{0}(\overline{\vec{u}} \cdot \vec{\nabla}) \psi=0 .
$$

\subsection{Loop quantum gravity}

In the nonperturbative approach to quantum gravity based on observables analogous to Wilson loops, the semi-classical gravitational field is described via expectation values in so-called "weave"states. Gambini and Pullin [9] discuss the propagation of light through a gravitational field represented by a parity-violating weave state and find a polarization dependence of light propagation inconsistent 
with the EEP. The weave state is characterized by the length scale $L$, and gives rise to effective Maxwell equations [9] of the form

$$
\begin{aligned}
\partial_{t} \boldsymbol{E} & =-\boldsymbol{\nabla} \times \boldsymbol{B}+2 \chi \ell_{\mathrm{P}} \boldsymbol{\nabla}^{2} \boldsymbol{B} \\
\partial_{t} \boldsymbol{B} & =\boldsymbol{\nabla} \times \boldsymbol{E}-2 \chi \ell_{\mathrm{P}} \nabla^{2} \boldsymbol{E} .
\end{aligned}
$$

A corresponding effective Dirac equation [38] has the form

$$
\left(i \widetilde{\gamma}^{a} \partial_{a}-\widetilde{m}+\widetilde{\gamma}^{a b} \partial_{a} \partial_{b}\right) \psi=0
$$

with $\widetilde{\gamma}^{a}=\gamma^{a}+\kappa_{1} \frac{\ell_{\mathrm{P}}}{L} G_{1}^{a}+\kappa_{2}\left(\frac{\ell_{\mathrm{P}}}{L}\right)^{2} G_{2}^{a}+\ldots, \widetilde{m}=m+\lambda_{1} \frac{\ell_{\mathrm{P}}}{L} M_{1}+\lambda_{2}\left(\frac{\ell_{\mathrm{P}}}{L}\right)^{2} M_{2}+\ldots$, and $\widetilde{\gamma}^{a b}=$ $\mu_{1} \frac{\ell_{\mathrm{P}}}{L} G_{1}^{a b}+\mu_{2}\left(\frac{\ell_{\mathrm{P}}}{L}\right)^{2} G_{2}^{a b}+\ldots$, where $\gamma^{a}$ are the usual Dirac $\gamma$-matrices, $m$ is the usual mass of the Dirac particle, $G_{i}^{a}, M_{i}$, and $G_{i}^{a b}$ are arbitrary matrices, $\ell_{\mathrm{P}}$ is the Planck length and the coefficients $\kappa_{i}, \lambda_{i}$ and $\mu_{i}$ are of the order unity $(i=1,2, \ldots)$.

Since these field equations feature second-order spatial derivatives they are no longer hyperbolic and clearly single out a preferred frame. In addition, note that equation (16) modifies the homogeneous Maxwell equations, disrupting the relationship between field and 4-vector potential!

\subsection{Gauge theories of gravity and other possibilities}

Gauge theories of gravity like the Poincaré gauge theory 39] that leads to a Riemann-Cartan geometry, or the gauge theory of a linear group that leads to a metric-affine theory [40], gives rise to additional gravitational fields like torsion and, in the latter case, to nonmetricity. If these additional fields couple directly to matter, they can break local Lorentz invariance by singling out a preferred frame as well as breaking local position invariance.

In supergravity theories, which gauge the super-Poincaré group, torsion emerges as a bilinear combination of fundamental spin- $\frac{3}{2}$-field, see reference [41, for example.

Though invented long ago, Kaluza-Klein theories arise as a low-energy limits of string theory, with all that that entails regarding the validity of the EEP, see reference [42], for example.

Finally, we note that nonsymmetric theories of gravity, like those devised by John Moffat, have been shown to predict departures from universal free fall and violations of local Lorentz invariance in the electromagnetic sector 443] and 44].

\section{Experimental and observational tests of the EEP}

In principle, the outcomes of almost any experiment of observation conducted in different gravitational environments could yield evidence of the breakdown of the EEP. There are, however, certain classes of experiments and observations that are sensitive to characteristic violations of the EEP revealed by analyses within the theoretical frameworks discussed in section 2 .

\subsection{Tests of the Universality of Freefall}

\subsubsection{Tests with bulk matter}

Experiments that search for composition-dependence of the freefall acceleration of macroscopic samples of matter are direct tests of the weak equivalence prinicple, one consequence of the EEP. It can be tested in traditional Eötvös fashion using torsion balance technology as in reference [5] or by monitoring the relative motion of freely falling bodies as in the Bremen drop tower experiment [45] and in the proposed MICROSCOPE [46] and STEP [6] space-based experiments. To date, the highest precision, of order $10^{-12}$, has been achieved by torsion balance experiments, but the STEP experiment is designed to reach a precision of $10^{-18}$. 
Equation (10) shows that macroscopic samples of spin-polarized matter may experience different gravitational accelerations than unpolarized matter. Torsion balance experiments looking for such differential accelerations have been conducted by by Ritter, Gillies and coworkers 47, 48]. They find no evidence of new spin-dependent forces.

\subsubsection{Tests with quantum particles}

As we saw in section 2, nonmetric theories of gravity can predict that quanta of different kinds fall with different accelerations in a gravitational field. Historically, there has been a great deal of interest in direct searches for such effects, especially in comparing the free fall acceleration of particles and antiparticles.

The first test of this type was performed by Witteborn and Fairbank 49 who tried to measure the gravitational acceleration of charged particles. A little later, Koester [50] showed that neutrons fall with the same way as classical bulk matter to an accuracy of a few percent. This result has been verified by means of neutron interferometry [51].

The potential for future matter interferometry tests of the EEP seems bright. Atomic interferometers have recently determined the gravitational acceleration toward the Earth to a part in $10^{9}$ and yield results consistent with the measured acceleration of bulk matter. Refinements of these devices are expected to produce still more precise results and can be used to search for spin-dependent accelerations like those in (10).

\subsection{Spectroscopic and atomic clock tests of the EEP}

As noted in preceding sections atoms are composites of the mass-energy of nucleons and electrons as well as of their electromagnetic and weak- and strong-interaction binding energies. Nonmetric theories whose matter-gravity couplings distinguish between these various contributions can cause not only gravitational accelerations that differ from atom to atom but also shifts in the energy spacings of atomic states that depend on an atom's velocity through or location in its gravitational environment. Spectroscopic and atomic clock experiments can search directly for these kinds of preferred-frame and preferred-location effects.

In Hughes-Drever-like experiments, for example, see Ref. [52], one searches for relative shifts between the frequencies of ground-state hyperfine transitions depending on atomic orientation in the gravitational environment. The interpretation of this type of experiment in the context of the $T H \epsilon \mu$-formalism is discussed in reference [14]. The interpretation in the context of the test theory of section 2.4 is discussed in reference [27].

Spectroscopic methods can also be used to search for the effects of spin-dependent and other EEP-violating effects predicted by equation (9), for example, see [53, 27]. These techniques have been used to verify the spin-rotation coupling [54, 55] in a search for anomalous spin-couplings [56].

Atomic clock technology is a particular, refined application of spectroscopic technique. Experiments that monitor the relative rates of different types of atomic clock for dependence on the clocks' velocity through or location in a gravitational field provide another kind of spectroscopic test of the EEP. In essence, such tests are either tests of relativistic Doppler shifts that are sensitive to the parameters $\alpha_{i j}$ in (2) or tests of gravitational redshifts that are sensitive to the parameters $\beta_{i j}$. The difficulty of moving clocks through large changes in gravitational potential or at speeds approaching that of light limits the precision of such experiments. However, the Gravity Probe A experiment [57] succeeded in imposing the constraint $\left|\beta_{i j}\right| \leq 10^{-4}$. A recent experiment employing trapped Lithium atoms moving at $6.4 \%$ of the speed of light [58] was able to impose the constraint $\left|\alpha_{i j}\right| \leq 10^{-6}$. Atomic clock technology has also been used to constrain EEP-violating time-dependence of the fine-structure constant [59]. 


\subsection{EEP tests involving observations of wave propagation}

Observations of the propagation of electromagnetic waves or other fields through a gravitational field are, in sense, a kind of experiment examing the effects of freefall. We discuss them separately, however, because of the distinctive way in which local effects that we think of as directly signalling violations of the EEP are allowed to build up as waves propagate over long distances.

Departures of the form of the Maxwell equations from their usual metric form induced by nonmetric couplings to gravity can lead to dispersive wave propagation or birefringence. Analogous departures of the Dirac equation from its usual metric form can also lead to dispersive propagation and make measurements of the arrival times of photons and neutrinos emitted from the same astrophysical event a test of EEP. The existence of very short duration events like supernova explosions and gamma ray bursts in combinations with the build up of gravitational delays over very great distances makes sharp tests possible.

Recently, limits on gravity-induced dispersion of electromagnetic wave propagation have been inferred from observations of quasars and gamma ray bursters 60. They constrain

$$
\frac{c_{\gamma}(\omega)-c_{\gamma}\left(\omega_{0}\right)}{c_{\gamma}\left(\omega_{0}\right)}, \quad \frac{c_{\gamma}^{+}-c_{\gamma}^{-}}{c_{\gamma}^{+}}
$$

where $c_{\gamma}\left(\omega_{0}\right)$ is the velocity of the photon for a given frequency $\omega$ or polarization \pm . Exploiting rapid time variation of gamma ray bursters, Schaefer [60] is able to to impose sharp constraints on gravityinduced dispersion, $\left|\left(c_{\gamma}(\omega)-c_{\gamma}\left(\omega_{0}\right)\right) / c_{\gamma}\left(\omega_{0}\right)\right| \leq 6 \times 10^{-21}$ for $\omega \sim 10^{18} \mathrm{~Hz}$ and $\omega_{0} \sim 10^{19} \mathrm{~Hz}$. See also 62 for implications of such data for quantum gravity models. Data constraining gravity-induced differences between the speed at which photons and neutrinos propagate are also imposed,

$$
\frac{c_{\gamma}^{ \pm}(\omega)-c_{\nu}^{ \pm}(\omega)}{c_{\gamma}^{ \pm}(\omega)}
$$

is also available.

Finally, observations that constrain differences between the speeds with which light with different polarizations propagates through a gravitational field have been analyzed [61] leading to the constraint $\left|\left(c_{\gamma}^{+}-c_{\gamma}^{-}\right) / c_{\gamma}^{+}\right| \leq 10^{-28}$.

\section{References}

[1] I. Newton: Philosophiae Naturalis Principia Mathematica (London 1686).

[2] A. Einstein: Jahrb. Radioact. Elect. 4, 411 (1907).

[3] L. Maleki: SpaceTime Mission: Clock Test of Relativity at four Solar Radii, this volume.

[4] R.V. Eötvös, V. Pekár and E. Fekete: Beiträge zum Gesetz der Proportionalität von Trägheit und Gravität, Ann. Physik 68, 11 (1922).

[5] Y. Su, B.R. Heckel, E.G. Adelberger, J.H. Gundlach, M. Harris, G.L. Smith, and H.E. Swanson: New test of the universality of free fall, Phys. Rev. D 50, 3614 (1994).

[6] N. Lockerbie, J. Mester, R. Torii, S. Vitale, and P. Worden: STEP: A Status Report, in C. Lämmerzahl, C.W.F. Everitt, F.W. Hehl (eds.): Gyrod, Clocks, Interferometers...: Testing Relativistic Gravity in Space, Springer-Velag 2001, p. 213.

[7] A. Einstein: Preuss. Akad. Wiss Berlin Sitzber. 688 (1916).

[8] J. Norton: General covariance and the foundations of general relativity: eight decades of dispute, Rep. Prog. Phys. 56, 791 (1993). 
[9] R. Gambini and J. Pullin: Nonstandard optics from quantum space-time, Phys. Rev. D 59, 124021 (1999).

[10] J. Ellis, N.E. Mavromatos and D.V. Nanopoulos: Probing models of quantum space-time foam, preprint gr-qc/9909085.

[11] M.P. Haugan and C. Lämmerzahl: On the experimental foundations of the Maxwell equations, Ann. Phys. (Leipzig) 9, 119 (2000).

[12] K. Nordtvedt: Qualitative relationship between clocks gravitational "red-shift" violatings and nonuniversality of free-fall rates in nonmetric theories of gravity, Phys. Rev. D 11, 245 (1975).

[13] M.P. Haugan: Energy Conservation and the Principle of Equivalence, Ann. Phys. (N.Y.) 118, 156 (1979).

[14] C.M. Will: Theory and Experiment in Gravitation Physics, revised edition (Cambridge University Press, Cambridge 1993).

[15] C.M. Will: The Confrontation between General Relativity and Experiment: A 1998 Update (Lecture notes from the 1998 SLAC Summer Institute on Particle Physics), gr-qc/9811036.

[16] M.P. Haugan and C.M. Will: Modern tests of special relativity, Physics Today, May 1987, p. 69.

[17] A.P. Lightman and D.L. Lee: Restricted Proof that the Weak Equivalence Principle Implies the Einstein Equivalence Principle, Phys. Rev. D 8, 364 (1973).

[18] C.M. Will: Gravitational red-shift measurements as tests of nonmetric theories of gravity, Phys. Rev. D 10, 2330 (1974).

[19] M.D. Gabriel and M.P. Haugan: Testing the Einstein Equivalence Principle: Atomic clocks and local Lorentz invariance, Phys. Rev. D 41, 2943 (1990).

[20] J.P. Turneaure, C.M. Will, B.F. Farrel, E.M. Mattison, and R.F.C. Vessot: Test of the principle of equivalence by a null gravitational red-shift experiment, Phys. Rev. 27, 1705 (1983).

[21] C. Alvarez and R.B. Mann: Testing the Equivalence Principle in the Quantum Regime, preprint (1996), Honorable mention in the Gravity Research Foundation Essay Contest (and references cited therein).

[22] J.E. Horvath, E.A. Logiudice, C. Riveros, and H. Vucetich: Einstein equivalence principle and theories of gravitation: A gravitationally modified standard model, Phys. Rev. D 38, 1754 (1988).

[23] W.-T. Ni: Equivalence Principles and Electromagnetism, Phys. Rev. Lett. 38, 301 (1977); Bull. Am. Phys. Soc. 19, 655 (1974); A Nonmetric Theory of Gravity, preprint, Montana State University, Bozeman, Montana, USA (1973), http://gravity5.phys.nthu.edu.tw.

[24] R.A. Puntigam, C. Lämmerzahl, and F.W. Hehl: Maxwell's theory on a post-Riemannian spacetime and the equivalence principle, Class. Qaunt. Grav. 14, 1347 (1997).

[25] S. Hojman, M.P. Rosenbaum, and L.C. Shepley: Gauge invariance, minimal coupling, and torsion, Phys. Rev. D 17, 3141 (1978).

[26] C. Lämmerzahl, Ch.J. Bordé: Testing the Dirac equation, in C. Lämmerzahl, C.W.F. Everitt, F.W. Hehl (eds.): Gyrod, Clocks, Interferometers...: Testing Relativistic Gravity in Space, Springer-Velag 2001, p. 466. 
[27] C. Lämmerzahl: Quantum Tests of Foundations of General Relativity, Class. Quantum Grav. 14, 13 (1998).

[28] N.D. Hari Dass: Test for $C, P$, and $T$ Nonconservation in Gravitaton, Phys. Rev. Lett. 36, 393 (1976).

[29] N.D. Hari Dass: Experimental Tests for Some Quantum Effects in Gravitation, Ann. Physics (N.Y.) 107, 337 (1977).

[30] A. Peres: Test of the equivalence principle with spin, Phys. Rev. D 18, 2739 (1978).

[31] D. Colloday and V.A. Kostelecky: CPT-violation and the standard model, Phys. Rev. D 55, 6760 (1997).

[32] D. Colloday and V.A. Kostelecky: Lorentz-violating extension of the standard model, Phys. Rev. D 55, 6760 (1997).

[33] T. Damour and A.M. Polyakov: The string dilaton and a least action principle, Nucl. Physics B 423, 532 (1994).

[34] T. Damour and A.M. Polyakov: String Theory and Gravity, Gen. Rel. Grav. 12, 1171 (1996).

[35] T. Damour: Equivalence Principle and Clocks, to appear in the Proceedings of the 34th Rencontres de Moriond, "Gravitational Waves and Experimental Gravity", January 1999, gr$\mathrm{qc} / 9904032$.

[36] J. Ellis, N.E. Mavromatos, and D.V. Nanopoulos: Probing models of quantum space-time foam, gr-qc/9909085.

[37] J. Ellis, N.E. Mavromatos, D.V. Nanopoulos, and G. Volkov: Gravitational-Recoil Effects on Fermion Propagation in Space-Time Foam, gr-qc/9911055.

[38] J. Alfaro, H.A. Morales-Tecotl, and L.F. Urrutia: Quantum gravity corrections to neutrino propagation, Phys. Rev. Lett. 84, to appear (2000).

[39] F.W. Hehl, P. von der Heyde, G.D. Kerlick, and J.M. Nester: General relativity with spin and torsion: Foundations and prospects, Rev. Mod. Phys. 48, 393 (1976).

[40] F.W. Hehl, J.D. McCrea, E.W. Mielke, and Y. Ne'eman: Metric-affine gauge theory of gravity: Field Equations, Noether Identities, World Spinors, and Breaking of Dilation Invariance, Phys. Rep. 258, 1 (1995).

[41] P. van Nieuwenhuizen: Supergravity, Phys. Rep. 68, 189 (1982).

[42] M.J. Duff: Kaluza-Klein theory in perspective, hep-th/9410046.

[43] C.M. Will, C.M.: Violation of the Weak Equivalence Principle in Theories of Gravity with a Nonsymmetric Metric, Phys. Rev. Lett. 62, 369 (1989).

[44] M.D. Gabriel, M.P. Haugan, R.B. Mann, and J.H. Palmer: Nonsymmetric gravitation theories and local Lorentz invariance, Phys. Rev. D 91, 2465 (1991).

[45] W. Vodel, H. Dittus, S. Nietzsche, H. Koch, J. v. Zameck Glyscinski, R. Neubert, S. Lochmann, C. Mehls, D. Lockowandt: High Sensitive DC SQUID Based Position Detectors for Application in Gravitational Experiments at the Drop Tower Bremen, this volume.

[46] P. Touboul: Space Accelerometers Present Status, in C. Lämmerzahl, C.W.F. Everitt, F.W. Hehl (eds.): Gyrod, Clocks, Interferometers...: Testing Relativistic Gravity in Space, SpringerVelag 2001, p. 274. 
[47] R.C. Ritter, C.E. Goldblum, W.-T. Ni, G.T. Gillies, and C.C. Speake: Experimental test of equivalence principle with polarized masses, Phys. Rev. D 42, 977 (1990).

[48] R.C. Ritter, L.I. Winkler, and G.T. Gillies: Search for Anomalous Spin-Dependent Forces with a Polarized-Mass Torsion Pendulum, Phys. Rev. Lett. 70, 701 (1993).

[49] F.C. Witteborn and W.M. Fairbank: Experimental Comparison of the Graviational Force on Freely Falling Electrons and Metallic Electrons, Phys. Rev. Lett. 19, 1049 (1967).

[50] L. Koester: Verification of the equivalence principle of gravitational and inertial mass for the neutron, Phys. Rev. D 14, 907 (1976).

[51] J.-L. Staudenmann, S.A. Werner, R. Colella, A.W. Overhauser: Gravity and Inertia in Quantum Mechanics, Phys. Rev. A 211419 (1980).

[52] T.E. Chupp, R.J. Hoara, R.A. Loveman, E.R. Oteiza, J.M. Richardson, and M.E. Wagshul: Results of a New Test of Local Lorentz Invariance: A Search for Mass Anisotropy in ${ }^{21} \mathrm{Ne}$, Phys. Rev. Lett. 63, 1541 (1989).

[53] C. Lämmerzahl: Constraints on space-time torsion from Hughes-Drever experiments, Phys. Lett. A 228, 223 (1997).

[54] B. Mashhoon: Neutron Interferometry in a Rotating Frame of Reference, Phys. Rev. Lett. 61, 2639 (1988).

[55] B. Mashhoon: On the coupling of intrinsic spin with the rotation of the earth, Phys. Lett. A 198, 9 (1995).

[56] B.J. Venema, P.K. Majumder, S.K. Lamoreaux, B.R. Heckel, and E.N. Fortson: Search for a Coupling of the Earth's Gravitational Field to Nuclear Spins in Atomic Mercury, Phys. Rev. Lett. 68, 135 (1992).

[57] R.F.C. Vessot, M.W. Levine, E.M. Mattison, E.L. Blomberg, T.E. Hoffmann, G.U. Nystrom, B.F. Farrel, R. Decher, P.B. Eby, C.R. Baughter, J.W. Watts, D.L. Teuber, and F.D. Wills: Test of Relativistic Gravitation with a Space-Borne Hydrogen Maser, Phys. Rev. Lett. 45, 2081 (1980).

[58] R. Grieser, R. Klein, G. Huber, S. Dickopf, I. Klaft, P. Knobloch, P. Merz, F. Albrecht, M. Grieser, D. Habs, D. Schwalm, and T. Kühl: A test of special relativity with stored lithium ions, Appl. Phys. B 59, 127 (1994).

[59] J.D. Prestage, R.L. Tjoelker, and L. Maleki: Atomic clocks and variations of the fine structure constant, Phys. Rev. Lett. 74, 3511 (1995).

[60] B.E. Schaefer: Severe limits on variations of the speed of light with frequency, Phys. Rev. Lett. 82, 4964 (1999).

[61] M.P. Haugan and T.F. Kauffmann: A New Test of the Einstein Equivalence Principle and the Isotropy of Space, Phys. Rev. D 52, 3168 (1995).

[62] S.D. Biller et al.: Limits to quantum gravity effects on energy dependence of the speed of light from observations of $\mathrm{TeV}$ flares in active galaxies, Phys. Rev. Lett. 83, 2108 (1999). 